\title{
Modelos explicativos de las funciones ejecutivas
}

\author{
Explanatory models of executive functions
}

\author{
Luis Miguel Echavarría ${ }^{1}$ \\ Universidad Tecnológica del Perú
}

Recibido: $11-05-17$

Aceptado: $01-06-17$

\section{Resumen}

Las funciones ejecutivas (FE) son consideradas como un constructo teórico, referido a la capacidad que tenemos como seres humanos para planificar, organizar, inhibir, monitorizar nuestra conducta y ser flexibles frente a situaciones novedosas. Sin embargo, dicha definición no es la única, ya que muchos autores la conceptualizan de diversas maneras y ello conlleva a que se presenten distintos modelos que tratan de explicar la naturaleza de tales funciones; no obstante, aún no se ha llegado a un consenso de la misma.

Por ello, el presente trabajo, pone a la luz aquellos modelos que buscan explicar las funciones ejecutivas en toda su magnitud y complejidad, encontrándose desde posturas anatómicas hasta tendencias estadísticas o considerar cómo se desarrollan las FE en las etapas del desarrollo, las mismas que fueron planteadas con el fin de tener un mejor entendimiento de ellas.

Palabras clave: Función ejecutiva, modelos explicativos, componentes.

\begin{abstract}
Executive functions (FE) are considered as a theoretical construct, referring to the capacity we have as human beings to plan, organize, inhibit, monitor our behavior and be flexible in the face of novel situations. However, this definition is not the only one, since many authors conceptualize it in different ways and this means that different models are presented that try to explain the nature of such functions; However, a consensus has not yet been reached. Therefore, the present work, brings to light those models that seek to explain executive functions in all their magnitude and complexity, finding from anatomical positions to statistical trends or to consider how they develop the FE in the stages of development, the same that were Raised in order to have a better understanding of them.
\end{abstract}

Keywords: Executive function, explanatory models, components.

\footnotetext{
1. Instituto de Atención Temprana e Investigación "Neuropsicología Infantil Perú". Universidad Tecnológica del Perú.Email: 1mer212@outlook.com 
Se considera a la neuropsicología como aquella ciencia que se encarga del estudio y la relación que se establece entre la actividad del sistema nervioso y el funcionamiento cognitivo/conductual de la persona, tanto en la normalidad como en la patología.

Siendo así, tuvo como objetivo clásico el de identificar las consecuencias del daño cerebral. En ese ínterin histórico, diversos han sido los casos clínicos que han servido como eje orientador de las actuales investigaciones y uno de los más resaltantes fue el de "Phineas Gage", un sujeto que tuvo un accidente en 1848 como consecuencia de que una barra de metal atravesase su cabeza, la misma que le generó alteraciones en su comportamiento y tal como lo mencionó el médico que lo atendió en esa época, Gage había perdido el equilibrio entre sus facultades intelectuales y sus propensiones animales.

Sin embargo, no se sabía por qué Phineas presentó tales manifestaciones y fue hasta 1992 en el que Hanna Damasio (citado en Damasio, 1994) en colaboración con el neurólogo Thomas Grabowski y el ingeniero Randall Frank, tuvieron la posibilidad de estudiar el cráneo de Gage mediante el análisis de las fotografías de éste que fueron previamente tomadas por el neurólogo Albert Galaburda, haciendo uso de un símil de computadora, logrando reconstruir la trayectoria de aquel proyectil que atravesó la cabeza de Phineas (Damasio, 1994). Tal es así que pudieron identificar que la barra de hierro afectó los lóbulos frontales, de ahí que se considera a Phineas Gage como el "caso prototípico de una pérdida de las FE".

Pero, ¿cómo se definen o que son las FE? Al respecto, podemos indicar que Luria fue uno de los precursores de la neuropsicología y quien en su planteamiento de sus "bloques funcionales" en el año 1974 (citado en Tirapu, 2009), hacía referencia a aquel encargado de la programación y control (tercer bloque funcional), el mismo que años más tarde fue acuñado por Muriel Lezak como "funciones ejecutivas", definiéndolas como aquellas capacidades para formular metas, planificar procesos y estrategias, ejecutar planes y aptitud para llevarlas de manera eficaz (Lezak, 1982). Sin embargo, autores como Shallice en 1988 (citado por Tirapu, 2009), las definió como aquellos procesos que relacionan ideas, acciones simples y movimientos, que orientan hacía la solución de situaciones consideradas complejas (Tirapu, 2009; Gonzáles, 2015). Así mismo, Sholberg y Mateer en el año 1989 (citados por Tirapu, 2009), asumieron que las FE abarcan un conjunto de procesos cognitivos, resaltando entre ellos: La elección de objetivos, la anticipación, la planificación, la selección de la conducta, el autocontrol, la autorregulación y el uso de la retroalimentación o feedback externo que recibe el sujeto (Tirapu, García, Luna, Roig y Pelegrín, 2008 A).

Por su parte Tirapu, García, Ríos y Ardila (2011), proponen que dicho constructo se refiere a la habilidad que poseemos para hallar soluciones frente a una situación problemática novedosa, siendo necesario para ello, el llevar a cabo una 
serie de predicciones o pronósticos de las posibles consecuencias a las que podemos llegar con cada una de las soluciones planteadas. Y así podemos encontrar otras definiciones, las cuales no logran llegar a un consenso, por lo cual es preciso hacer una revisión por todas aquellas propuestas que tratan de explicar a las FE.

\section{MÉTODO}

Se hizo una revisión bibliográfica del concepto de FE y se identificaron diversos modelos que explicaban su definición y cuales eran aquellas funciones que la componían. Dichos modelos estudiaron poblaciones clínicas, se encontró aquellos que aplicaron técnicas de neuroimagen o técnicas estadísticas (Gonzáles, 2015), y aquellos que se interesaron por su estudio en diversas etapas del desarrollo (Roselli, Jurado y Matute, 2008; Gonzáles, 2015) con el fin de comprender y sustentar su entendimiento y, además, permitirán conocer los distintos componentes de las FE.

\section{RESULTADOS}

Hablar de FE, implica tratar los lóbulos frontales y es que éstos constituyen la estructura crítica en el desarrollo del sistema nervioso, cuya función, anatomía y conexiones ha sido objeto de innumerables estudios a lo largo de los últimos años, aunque muchos de los procesos en los que se encuentra involucrado están aún por definir (Tirapu, García, Ríos y Ardila, 2011).

Cabe indicar que los procesos neuropsicológicos que poseen los lóbulos frontales son muy diversos y numerosos, siendo alguno de ello: El control y programación motora, el control atencional y la memoria, la cognición social y la metacognición (Flores y Ostrosky, 2012). Aunque, algunas investigaciones indican que la actividad principal de los lóbulos frontales corresponde a las funciones del proceso de atención, lo que Luria consideró como su tercer bloque cerebral o funcional (encargado de la programación, control y verificación) (Echavarría, 2013).

Al hablar de componentes de las FE, necesariamente, se deben detallar los diversos modelos explicativos de éstas (Flores, 2006; Flores y Ostrosky, 2012; Portellano y García, 2014; Tirapu et al, 2011, Gonzáles, 2015):

Bloques Funcionales (1974): Entre la Segunda Guerra Mundial y los años posteriores a la misma, el número de sujetos que fueron heridos en circunstancias de conflicto y que presentaban alteraciones cognitivas, resultado de lesiones cerebrales, tuvo un incremento significativo, así como también la necesidad de contar con procedimientos diagnósticos y de rehabilitación para ellos.

Si bien es conocido que Luria (citado en Tirapu, 2009) en ningún momento planteó o usó el término de "funciones ejecutivas", fue el primer referente al estudiar un grupo de pacientes con lesiones frontales e indicar que los lóbulos frontales se relacionan con las 
capacidades de programación, regulación y verificación de la conducta, ello en referencia a la propuesta teórica de las "Tres unidades o bloques funcionales".

Se acuña el término "función ejecutiva" (1982): Muriel Lezak (citado en Tirapu, 2009), estudió un grupo de pacientes con lesiones frontales y los hallazgos que obtuvo fue que dicha población presentaba alteraciones en la iniciativa y motivación, incapacidad de plantear metas y objetivos (Flores y Ostrosky, 2012). De ahí surgió la propuesta de indicar que las FE son las encargadas de la formulación de metas, planeación, mantenimiento y realización del plan.

\section{Paradigmas de supervisión atencional orientada a objetivos:}

* Modelo de procesamiento de la información: Norman y Shallice en el año 1986 (citados en Miller \& Cummings, 2007), luego de estudiar una muestra de pacientes con lesiones frontales y una población normal, hicieron la propuesta del Sistema Atencional Supervisor representado en la corteza prefrontal (CPF), en la que señalan que el comportamiento humano se mediatiza por ciertos esquemas mentales que especifican la interpretación de las entradas o inputs externos y la subsiguiente acción o respuesta (Flores \& Ostrosky, 2012).

* Teoría integradora de la corteza prefrontal: Earl Miller y Jonathan Cohen (citados en Miller \& Cummings, 2007), indican que la corteza prefrontal desempeña un papel destacado en el mantenimiento de pautas de actividad que representan objetivos y los medios para conseguirlos.

* Modelo de control atencional: Los autores de este modelo fueron Stuss y colaboradores (citados en Tirapu et al., 2011), quienes propusieron siete funciones atencionales que tenía su respectiva correlación anatómica: Mantenimiento (frontal derecho), supresión (prefrontal dorsolateral), alternancia (prefrontal dorsolateral y frontal medial), preparación (prefrontal dorsolateral), atención dividida (cingulado y orbitofrontal), concentración (cingulado) y programación (prefrontal dorsolateral).

* Hipótesis del filtro dinámico: En el 2000 Shimamura propone que la corteza prefrontal es la responsable de controlar y monitorizar la información, procesándola a través de un proceso de filtrado, el cual se constituye por cuatro aspectos: Selección, mantenimiento, actualización y redirección (Flores y Ostrosky, 2012).

\section{- Modelos de constructo único:}

* Hipótesis de la información contextual: Jonathan Cohen y sus colaboradores en la época de los noventa, propusieron esta teoría al entender que el contexto es un componente importante para entender las alteraciones ejecutivas 
observadas en sujetos con esquizofrenia. Al respecto señalan que dichos pacientes muestran deterioro ejecutivo como consecuencia de la dificultad que tienen para mantener, representar o actualizar la información de su medio.

El postulado al que llegan es que diferentes procesos cognitivos (atención, memoria de trabajo o inhibición, por ejemplo), implicados en el control cognitivo, son en sí el reflejo de un solo y único mecanismo que opera bajo circunstancias diferentes.

* El factor "g" y el factor "I": En su teoría bifactorial de la inteligencia, Spearman proponía que la ejecución de cualquier actividad mental depende de dos factores diferenciados (Tirapu et al., 2011) y estos son:

- Factor general "g": es la base común de la inteligencia que se mantiene igual en cuanto a las capacidades relacionadas entre sí, pese a que varían libremente de un vínculo a otro.

- Factor específico "s": son aquellas competencias específicas que varían de un individuo a otro y también de una capacidad a otra.

Por su parte, John Duncan planteó que la inteligencia es una propiedad que emerge de una función localizada en una zona bien delimitada del cerebro: la CPF lateral y no es una propiedad emergente del conjunto del cerebro (Lezak, Howieson, Bigler \& Tranel, 2012; Flores y Ostrosky, 2012).

De otro lado, Diamond (citada en Flores y Ostrosky, 2012), encontró que existe relación entre las FE y la inteligencia cristalizada la cual hace referencia a la capacidad intelectual aprendida, pero halló que existe una mayor relación entre las FE y la inteligencia fluida que alude a la capacidad de solucionar problemas y razonamiento.

* Memoria de trabajo (MT): Baddeley (1992) planteó que las personas presentamos una capacidad que nos permite mantener mentalmente una determinada información mientras realizamos una actividad o resolvemos un problema (Tirapu et al., 2008 A; Tirapu et al., 2011).

Dicho modelo supone la existencia de un sistema de atención que se encargue de controlar a otros sistemas dependientes, el cual en su conjunto es denominado "ejecutivo central" o administrador central. Éste se encuentra encargado del procesamiento ejecutivo, que incluye: Dirigir la atención hacía la información relevante, suprimir la información irrelevante y las acciones indeseadas, supervisar la integración de información, coordinar múltiples procesos cognitivos que se desarrollan en paralelo y coordinar los subsistemas de la MT; de otro lado, los sistemas subordinados son el bucle fonológico y la agenda viso-espacial, ambos responsables del mantener de manera temporal la información (Baddeley, 1992; 
Tirapu y Céspedes, 2005; Flores y Ostrosky, 2012). En la misma obra se menciona que, Goldman-Rakic en 1987, propuso que la CPF es esencial para la MT, haciendo referencia al modelo de Baddeley respecto del mantenimiento a corto plazo de la información que es importante para una tarea.

Cabe precisar que, en diversas revisiones bibliográficas, se plantea que la MT ha evolucionado en cuanto a conceptualización, puesto que inicialmente se le consideraba como una memoria a corto plazo, mientras que algunos autores indican que es un sistema multicomponentes (Musso, 2009).

\section{- Modelos de secuenciación temporal:}

* Teoría representacional: Sugiere que la CPF tiene como función principal el manipular la información que se encuentra almacenada en otras regiones corticales y también de aquella disponible en estructuras subcorticales.

* CPF y organización temporal de la conducta: Fuster señaló como postulado que el rol más importante de la CPF es la de estructurar temporalmente la conducta, para lo cual se sirve de cuatro mecanismos fundamentales: Control inhibitorio, set preparatorio, memoria operativa, mecanismo de supervisión (Tirapu et al., 2011, Flores \& Ostrosky 2012).

\section{- Modelos jerárquicos - funcionales de la CPF:}

* Modelo del eje rostrocaudal de la CPF: Kalina Christoff y colaboradores, platearon que la CPF se encuentra estructurada funcionalmente de manera jerárquica, para lo cual parten de la hipótesis de que los procesos de razonamiento se basan en la manipulación de información en diversos niveles de complejidad (Tirapu et al., 2011).

* Modelo de la puerta de entrada (Gateway hypothesis): Formulada por Paul Burges (citado por Tirapu et al., 2008B) para indicar que el polo frontal es la estructura clave en la cognición, la misma que se orienta hacía estímulos ambientales o externos al cuerpo, para generar planes y mantenerlos a través de la reflexión (Tirapu et al., 2011).

* Modelo funcional en cascada de la CPF: Etienne Koechlin (citado por Tirapu et al., 2011), postuló dicha hipótesis para explicar la manera por la cual la CPF mantiene a las funciones complejas de manera diferenciada, para ello se basó en dos ejes: Anterior - posterior y medial - lateral.

\section{- Modelos integradores "emoción - cognición":}

* Marcador somático: Fue postulada por Damasio (citado por Bruna, Roig, Puyuelo, Junqué y Ruano, 2011), luego de hacer algunas observaciones en pacientes con lesiones frontales con el fin de tratar de explicar cuáles eran las 
zonas de la CPF implicadas en los procesos de razonamiento y toma de decisiones. Luego de analizar sus resultados, precisó que los pacientes con los que trabajó, presentaban dificultades en el funcionamiento diario y además mostraban severas alteraciones en el dominio personal y social; sin embargo, mantenían conservados el razonamiento, la toma de decisiones, la capacidad intelectual, el lenguaje, la MT o la atención básica (Bruna et al., 2011)

Ahora bien, respecto al marcador somático, fue postulado con el objetivo de explicar de qué manera influyen las emociones sobre la toma de decisiones y el razonamiento, llegándose a la conclusión que éstos últimos, dependen de variados niveles de operaciones neurobiológicas, dentro de las cuales algunas son solamente cognitivas y otras no; además, todas las operaciones mentales son dependientes de algún proceso básico como la atención y la MT (Bruna et al, 2011; Tirapu et al, 2011).

* Hipótesis de la complejidad cognitiva y control: Fue propuesta por Philip Zelazo, quien señaló que el desarrollo de las FE en la infancia, implica la aparición de distintas capacidades cognitivas que deben permitir al niño: Autorregular su conducta para lograr actuar de forma reflexiva y no impulsiva; mantener información, manipularla y actuar en función de ella, así como adaptar su comportamiento a los cambios que pueden producirse en el entorno (Tirapu et al., 2011).

- Modelos que emplearon técnicas estadísticas: Se hará referencia a aquellas teorías que usaron el análisis factorial para explicar los componentes de las FE.

* Tres factores independientes: Miyake y colaboradores (citados por Tirapu et al., 2008A), hicieron referencia a tres componentes ejecutivos muy bien diferenciados, pero que no mantienen total independencia para indicar que las FE se encuentra compuestas por: Actualización, inhibición y alternancia (Tirapu et al., 2008A; Tirapu et al., 2011; Gonzáles, 2015).

* Tres factores ejecutivos: Kyle Boone y colaboradores (citados por Tirapu et al., 2011), luego de someter a un análisis una muestra heterogénea de sujetos con alteraciones neurológicas, identificaron tres factores ejecutivos: 1) velocidad de procesamiento y atención básica y dividida, 2) flexibilidad cognitiva, y 3) memoria de corto plazo (Tirapu et al., 2008A; Tirapu et al., 2011).

* Tres factores: Robyn Busch y colaboradores (citados por Tirapu et al., 2011), en una muestra de pacientes con traumatismo craneoencefálico, mediante el análisis factorial, lograron identificar tres componentes que explicaría como está compuesta la FE: i) Primer factor que incluye FE de alto nivel (considera dos componentes: flexibilidad cognitiva y conducta autogenerada), ii) segundo factor (MT, encargada al parecer del control cognitivo) y el iii) tercer factor (parece tener relación con el control inhibitorio, puesto que en dicho factor, se consideran 
los errores cometidos al intentar inhibir la información inadecuada, lo cual es indicador de una falla en la memoria).

* Tres componentes: John Taylor y colaboradores señalan tres componentes generales relacionados con la función de la CPF: Establecimiento de uniones entre representaciones en la MT; la creación, estudio y decisión entre esquemas de alto nivel que incorporan secuencias de acción que se pueden repetir, pero a menudo suelen ser flexibles; además, evaluaciones afectivas que se incorporan utilizando éstas para dirigir acciones (Tirapu et al., 2008 A; Tirapu et al., 2011).

* Cuatro factores: Marcos Ríos y colaboradores en un grupo de pacientes con daño cerebral, llevaron a cabo un análisis factorial en una serie de tareas en las que se encontraban implicados los procesos de atención y control ejecutivo. Dicho trabajo les permitió identificar los siguientes factores de la función ejecutiva: 1) velocidad de procesamiento, 2) flexibilidad cognitiva, 3) memoria operativa y 4) control de la interferencia (Tirapu et al., 2011).

* Cinco factores independientes: Anderson en el 2001 (citado en Gonzáles, 2015), propuso que las FE presentan tres factores independientes y luego de analizar una población normal de 11 a 17 años, mediante el análisis de componentes principales con rotación Varimax, identificó que dentro de las FE es posible identificar 5 factores independientes, pero no correlacionados.

- Modelo conceptual: Julio Flores y colaboradores plantean dicho modelo, el mismo que se divide en cuatro niveles jerárquicos. En el menor nivel, se logran encontrar las funciones frontales básicas (control inhibitorio, control motor, detección de selecciones de riesgo); en el segundo nivel se encuentra el sistema de memoria; en tanto que en el tercer nivel se encuentran las funciones ejecutivas (planeación, fluidez, productividad, secuenciación, flexibilidad mental, eficiencia, generación de hipótesis de clasificación, entre otras); mientras que en el nivel más complejo se hallan las metafunciones (metacognicicón, abstracción y comprensión del sentido figurado) (Flores \& Ostrosky, 2012).

\section{- Modelos basados en diferentes etapas del desarrollo:}

* En el 2002 Anderson (citado en Gonzáles, 2015), evaluó en diferentes etapas del desarrollo (infancia tardía y adolescencia), el control atencional, la flexibilidad cognitiva y el establecimiento de metas. Sus hallazgos, permitieron encontrar patrones diferentes entre los 7 y 17 años.

* De Luca, Wood, Anderson, Buchanan, Proffitt, Mahony y Pantelis, en el 2003 en una muestra de diferentes etapas del desarrollo (infancia tardía hasta la vejez), usando la Batería Automatizada de Prueba Neuropsicológica de Cambridge (CANTAB) evaluaron la flexibilidad mental y la planeación. Los resultados de la FE en el grupo de niños de 8 años de edad se ampliaron, con ganancias funcionales 
encontradas en la eficacia de la capacidad de MT, la planificación y la capacidad para resolver problemas, entre las edades de 15 y 19 años y de nuevo a los 20-29 años de edad. La flexibilidad cognitiva se evaluó a nivel de adultos, incluso en los niños más pequeños. Se apreció un descenso en el desempeño en todas las actividades para la muestra de 50 a 64 años, lo que respalda la vulnerabilidad de las habilidades ejecutivas al envejecimiento normal.

* En el 2004 Brocky \& Bohlin (citados en Gonzáles, 2015; Roseelli, Jurado $\&$ Matute, 2008), evaluaron en una muestra de infantes la inhibición, velocidad de procesamiento y MT. Los resultados fueron que existen 3 períodos de maduración de las FE: a los 3, 9 y 14 años.

* También en el 2004 Gestard, Jerard \& Diamond (citados en Gonzáles, 2015), realizaron una evaluación, en la infancia temprana y tardía, del procesamiento riesgo - beneficio, encontrando que existe un incremento continuo.

\section{CONCLUSIONES}

Como puede observarse en lo señalado anteriormente, las FE son el resultado de un combinación y convergencia de diversas capacidades cognitivas, las mismas que no se encuentran lo suficientemente especificadas, tanto a nivel conceptual como metodológico (Stuss y Anderson, 2004; Rabbit, 1998, citados por Flores y Ostrosky, 2012); por lo tanto, no son una entidad simple (Tirapu et al, 2008 B).

Los modelos, hipótesis o teorías descritas de manera sucinta anteriormente, permiten identificar que existen distintas formas de abordar el estudio de las FE, realizando aproximaciones a este constructo teórico con posturas diversas, por un lado aquellas basadas en la adaptabilidad (como el modelo propuesto por Duncan) en el que se considera que los lóbulos frontales desempeñan funciones de supervisión inespecíficas que se adaptan a una gran variedad de tareas, o aquellas en las que asocia diferentes procesos ejecutivos con distintas regiones frontales (como en el modelo formulado por Stuss) o aquellas en las que se pone en juego la relación entre las emociones y los procesos cognitivos, o en los que se toma importancia al análisis factorial o estadístico o en los estudios llevados a cabos en distintas poblaciones en función a las etapas del desarrollo. Es preciso indicar que los planteamientos realizados por los distintos autores no se confrontan, ni tampoco se contradicen, sino más bien y, por el contrario, se complementan entre sí.

Además, de ello, en la mayoría de estos modelos se pueden identificar diversos componentes de las FE, llegándose a concluir que todos ellos facilitan la anticipación y el establecimiento de metas, permitiendo formar planes, iniciar las actividades, autorregularse y alcanzar la habilidad para llevarlas a cabo de manera eficaz; además, el acuerdo que se tiene es que estas funciones vitales para los seres humanos (Tirapu et al., 2008 A; Tirapu et al., 2011) y que permiten al individuo 
controlar y regular su conducta mediante diversos procesos cognitivos (Tirapu et al, 2008 B).

Finalmente, diremos que todos los autores e investigaciones, si bien no llegan a un consenso sobre qué son las FE, sí asumen que ellas son las capacidades de máximo desarrollo en nuestra especie e incluso se llega a concluir que son exclusivas de los seres humanos (Rivera, 2013), y que nos permiten desenvolvernos de manera organizada en nuestra vida diaria, otorgándoles la condición de estar implicadas en la mayoría, por no decir en todas, las capacidades cognitivas, transformando el pensamiento en acción y regulando las mismas.

\section{REFERENCIAS}

Badelley, A. (1992). Working memory. Science, 255, 556 - 559. Recuperado de: https:// www.cs.indiana.edu/ port/HDphonol/Baddely.wkg.mem.Science.pdf

Bruna, O., Roig, T., Puyuelo, M., Junqué, C. \& Ruano, Á. (2011). Rehabilitación neuropsicológica: Intervención y práctica clínica. Barcelona: Elseiver Masson.

Damasio, A. (1994). El error de Descartés: La razón de las emociones. Santiago de Chile: Andrés Bello.

De Luca, C., Wood, S., Anderson, V., Buchanan, J., Proffitt, T., Mahony K. \& Pantelis, C. (2003). Normative data from the CANTAB. I: development of executive function over the lifespan. Journal of clinical and experimental neuropsychology, 25(2), 242 - 254. Doi: 10.1076/jcen.25.2.242.13639

Echavarría, L. (2013). El proceso de la atención: una mirada desde la neuropsicología. Revista Digital EOS Perú, 1(1), 15 - 18. Recuperado de: http://eosperu.net/revista/ wp-content/uploads/2015/10/EL-PROCESO-DE-LA-ATENCI\%C3\%93N-UNAMIRADA-DESDE-LA-NEUROPSICOLOG\%C3\%8DA.pdf

Flores, J. (2006). Neuropsicología de Lóbulos Frontales. México: Universidad Juárez Autónoma de Tabasco.

Flores, J. y Ostrosky, F. (2012). Desarrollo neuropsicológico de lóbulos frontales y funciones ejecutivas. México: Manual Moderno.

Goldman - Rakic, P. (1987). Development of cortical and cognitive function. Child Development 58(3), 601 - 622. Recuperado de: http://www.jstor.org/stable/1130201

Gonzáles, M. (2015). Desarrollo neuropsicológico de las funciones ejecutivas en preescolar. México: Manual Moderno.

Lezak, M. (1982). The problem of assessing Executive Functions. International Journal of Psychology, 17, 281 - 297. Recuperado de: http://onlinelibrary.wiley.com/ doi/10.1080/00207598208247445/pdf

Lezak, M., Howieson, D., Bigler, E. \& Tranle, D. (2012). Neuropsychological assessment. 5a ed. Oxford: University Press.

Miller, B. \& Cummings, J. (2007. The Human Frontal Lobes: functions and disorders. 2a ed. New York: The Guilford Press. 
Musso, M. (2009). Funciones ejecutivas y control ejecutivo: una revisión bibliográfica mirando la arquitectura de la mente. Revista de Psicología, 5(9), 106 - 123. Recuperado de: http://bibliotecadigital.uca.edu.ar/repositorio/revistas/funcionesejecutivas-control.pdf

Portellano, J. y García, J. (2014). Neuropsicología de la atención, las funciones ejecutivas y la memoria. Madrid: Editorial Síntesis.

Rivera, A. (9 de agosto de 2013). Funciones ejecutivas [Entrada en un blog]. Psicobiología del género Homo. Recuperado de: http://psicobiologiadelgenerohomo.blogspot. pe/2013/08/funciones-ejecutivas.html

Rosselli, M.; Jurado, M. y Matute, E. (2008). Las funciones ejecutivas a través de la vida. Revista Neuropsicología, Neuropsiquiatría y Neurociencias, 8(1), 23 - 46. Recuperado de: http://neurociencias.udea.edu.co/revista/PDF/REVNEURO_vol8_ num1_6.pdf.

Shimamura, A. (2000). The role of the prefrontal cortex in dynamic filtering. Psychobiology, 28(2), 207 - 218. Recuperado de: http://socrates.berkeley.edu/ shimlab/2000 Shimamura_DynFilter.pdf

Tirapu, J. y Céspedes, J. (2005). Memoria y funciones ejecutivas. Revista de Neurología, 41(8), 475 - 484. Recuperado de: http://www.uma.es/media/files/Memoria_y_ funciones_ejecutivas.pdf

Tirapu, J., García, A., Luna, P., Roig, T. y Pelegrín, C. (2008 A). Modelos de funciones y control ejecutivo (I). Revista de Neurología, 46 (11), 684 - 692. Recuperado de: http:// www.neurologia.com/articulo/2008119

Tirapu, J., García, A., Luna, P., Roig, T. y Pelegrín, C. (2008 B). Modelos de funciones y control ejecutivo (II). Revista de Neurología, 46 (12), 742 - 750. Recuperado de: https://www.psyciencia.com/wp-content/uploads/2012/10/modelos-de-funciones-ycontrol-ejecutivo-II.pdf

Tirapu, J. (2009). Estimulación y rehabilitación cognitiva de las funciones ejecutivas. En: Muñoz, E., Blázquez, J., Galpasoro, N., Gonzáles, B., Lubrini, G., Periáñez, J., Ríos, M., Sáncehz, I., Tirapu, J. \& Zulaica, A. Estimulación cognitiva (módulo 7: p. 1 - 73). Barcelona: Eureca Media.

Tirapu, J., García, A., Ríos, M. y Ardila, A. (2011). Neuropsicología de la corteza prefrontal y las funciones ejecutivas. España: Viguera Ediciones. 
